\title{
Les modifications de la ligne de rivage dans les îles de la Société (Polynésie française) : un indicateur des pressions anthropiques en zone côtière
}

Caroline Vieux, Yannick Chancerelle, Annie Aubanel et Bernard Salvat

\section{(2) OpenEdition Journals}

Édition électronique

URL : http://journals.openedition.org/jso/3162

DOI : $10.4000 /$ jso.3162

ISSN : $1760-7256$

Éditeur

Société des océanistes

Édition imprimée

Date de publication : 15 décembre 2008

Pagination : 59-66

ISBN : 978-2-85430-012-3

ISSN : 0300-953x

Référence électronique

Caroline Vieux, Yannick Chancerelle, Annie Aubanel et Bernard Salvat, « Les modifications de la ligne de rivage dans les îles de la Société (Polynésie française) : un indicateur des pressions anthropiques en zone côtière », Journal de la Société des Océanistes [En ligne], 126-127 | Année 2008, mis en ligne le 15 décembre 2011, consulté le 17 juin 2020. URL : http://journals.openedition.org/jso/3162 ; DOI : https://doi.org/10.4000/jso.3162 


\title{
Les modifications de la ligne de rivage dans les îles de la Société (Polynésie française) : un indicateur des pressions anthropiques en zone côtière
}

\author{
par
}

Caroline VIEUX*, Yannick CHANCRELLE*, Annie AUBANEL**

et Bernard SALVAT*

\section{RÉSUMÉ}

Dans les îles océaniques tropicales, la ligne de rivage naturelle correspond à une plage, à une falaise, à des galets ou rochers, à des zones de végétation basse ou haute. Le développement économique des îles et la croissance démographique de leurs populations ont pour conséquence une artificialisation de cette ligne de rivage pour utilité publique ou par des riverains qui cherchent à se protéger des actions de la mer, mais aussi à étendre leur emprise sur le lagon. Plusieurs îles de la Société ont fait l'objet d'une étude quantifiée de leurs lignes de rivage afin de déterminer leurs degrés d'anthropisation. Dans plusieurs d'entre elles, cette artificialisation est voisine ou dépasse $40 \%$ du linéaire côtier. Elle est un bon indicateur des pressions anthropiques en zone côtière. L'évolution de cette tendance a pu être montrée à Moorea par des études menées à quinze ans d'intervalle. Des actions sont entreprises et doivent être développées pour mettre fin cette artificialisation aux conséquences néfastes pour l'environnement.

MoTS-CLÉs : rivage, côtier, récifs, lagons, dégradations

La ligne de rivage est définie comme la limite entre le domaine terrestre et le domaine maritime par hautes mers de vives-eaux qui marque la limite administrative des 50 pas géométriques.

\begin{abstract}
In tropical oceanic islands, coastlines are usually either sandy, rocky or have cliffs, - or may have low or high vegetation. Developing island states with increasing human populations are modifying coastlines to form artificial or modified structures for public utility or by lagoonside residents hoping to protect their property from the sea or to gain extra land on the margins of the lagoon. Several French Society Islands have been examined to establish the extent of this coastal modification. In most of them, human activities have altered $40 \%$ or more of the coastline, and the percentage of modification is a good indicator of the human pressure on the shoreline. The evolution of coastal modification has been documented on Moorea island during two field studies separated by a fifteen year period. Specific activities need to be developed and deployed to stop coastal modifications that result in damage to the environment.
\end{abstract}

KeYwords: Shoreline, coastal, reefs, lagoons, degradations

Elle se présente en milieu tropical comme une plage de sable, une falaise, une zone de rochers, une mangrove soit comme une frontière artificielle sous la forme d'un quai, d'un mur de sou-

\footnotetext{
* Centre de recherches insulaires et Observatoire de l'Environnement, Moorea, Polynésie française, ums 2978 CNRS-EPHE et UMR 5244 CNRS-EPHE-UPVD, université de Perpignan, bsalvat@univ-perp.fr, criobe@mail.pf

** Service de l'Urbanisme, IFRECor Polynésie, Papeete, aubanel@mail.pf
} 
tènement ou une d'une route bordière. À l'échelle géologique, la ligne de rivage se déplace et se modifie de façon naturelle selon les deux phénomènes géologiques: 1 . les mouvements verticaux du continent ou de l'île qui font varier la ligne de rivage et dans le cas de la subsidence la font remonter vers les terres et diminuent ainsi la surface des terres émergées; 2 . les variations du niveau de la mer qui font reculer vers les terres ou avancer vers la mer la ligne de rivage. En période stable, le rivage est continuellement alimenté par des apports de matériaux issus de la destruction mécanique du récif corallien par les vagues et par les organismes bio-érodeurs (organismes foreurs et brouteurs de calcaire) ainsi que par des apports de matériaux terrigènes issus de l'érosion des versants des îles hautes. Par ailleurs, les actions hydrodynamiques continuelles ou exceptionnelles (cyclones, tempêtes) engendrent selon les cas des érosions ou des engraissements de plage.

Depuis la fin de la dernière période glaciaire (18 000 ans BP), le niveau de la mer s'est relevé de plus d'une centaine de mètres à une vitesse de l'ordre de 1 à $3 \mathrm{~cm}$ par an au début de son élévation, puis à des vitesses variables, mais plus faibles par la suite (Paskoff, 1994). Depuis un siècle, l'élévation du niveau marin, variable selon les régions, a été d'environ $1,5 \mathrm{~mm}$ par an soit 15 $\mathrm{cm}$ au total. Cette élévation correspondant à une augmentation de la température moyenne de $0,5^{\circ} \mathrm{C}$ est due essentiellement à la régression des glaciers de montagne et à une diminution de la densité de l'eau océanique à la suite de son réchauffement. Nous sommes donc dans une phase d'érosion de la mer qui provoque un démaigrissement et un recul des plages (Grellet et al., 1993).

Parallèlement à cette évolution naturelle, le profil et la nature de la ligne de rivage peuvent se trouver modifiés directement par l'homme. C'est le phénomène d'anthropisation ou d'artificialisation qui atteint alors la ligne de rivage et qui consiste en la mise en place de remblais en terre, ou de béton ou de rochers, à des fins diverses. Cette artificialisation de la ligne de rivage, objet de notre étude dans les îles de la Société en Polynésie française, est plus particulièrement étudiée en métropole et dans les pays développés. Elle englobe alors non seulement l'éventuelle modification de la ligne de rivage mais aussi les implantations humaines situées en arrière de celle-ci.

La Polynésie française, dont la population a quintuplé depuis la fin de la Deuxième Guerre mondiale, se concentre sur un cordon littoral étroit dans les îles hautes, à l'interface des milieux lagonaire et terrestre sans considérer les motus éventuellement présents sur le récif barrière. Ce territoire n'échappe pas au phénomène d'anthropisation de sa ligne de rivage dans certaines îles. La concentration des activités humaines s'est fait porter tout particulièrement sur le littoral de l'île de Tahiti mais aussi sur les îles proches de Moorea, de Raiatea et de Bora-Bora dans l'archipel des îles de la Société. L'accroissement de la population en Polynésie française ainsi que le développement économique étant concentrés sur un nombre restreint d'îles, il paraît intéressant d'analyser l'état actuel du linéaire de côte de ces îles.

Notre analyse s'appuie sur des études réalisées à la fin des années 1980, à Huahine tout d'abord (Gabrié, 1988) puis à Bora-Bora (Galzin et al., 1990). Ces études insistent sur les conséquences écologiques et socio-économiques néfastes de travaux de remblaiements et d'extraction de matériaux coralliens dans des zones frangeantes. Ces travaux sont les principaux agents de la pression humaine sur la ligne de rivage. Nous considérerons ensuite les travaux réalisés dans les années 1990 qui furent des études quantitatives destinées à mesurer des pourcentages de linéaire côtier de diverses catégories de la ligne de rivage : Aubanel et al. (1991) et Aubanel (1993) sur l'île de Moorea, Aubanel et al (1999) et Marquet (1994) dans les îles-Sous-le-Vent, Polti (2001) et Sinjound (2002) à nouveau sur Moorea et enfin Leille (2005) et Raynal (2004) sur Tahiti. Ces études réalisées entre 1993 et 2005 nous ont permis de caractériser la nature de la ligne de rivage des îles de La Société, mais aussi de mesurer l'évolution de l'une d'entre elles dans le temps, Moorea. Nous discuterons enfin des conséquences de cette occupation humaine des côtes et des solutions envisagées pour contrôler l'évolution de la ligne de rivage et assurer une protection de la zone littorale face au développement des activités humaines.

\section{Matériel et méthodes}

La Polynésie française regroupe 118 îles essaimées dans la zone intertropicale du Pacifique Sud. Elle est divisée en cinq archipels (Marquises, Tuamotu, Australes, Gambier, Société) répartis sur une superficie océanique de 5 millions de $\mathrm{km}^{2}$. L'archipel de La Société comprend les îles du Vent dont Tahiti, la plus grande des îles $\left(1042 \mathrm{~km}^{2}\right)$, Moorea $\left(136 \mathrm{~km}^{2}\right)$ et les îles Sous-le-Vent dont Bora Bora, la plus touristique des îles. Le recensement de la population en Polynésie française pour l'année 2002 est de 
241800 habitants. L'archipel de la Société rassemble à lui seul $87 \%$ de la population.

Pour caractériser la ligne de rivage des îles hautes, une même méthode a été utilisée dans toutes les études conduites dans les îles de la Société (Aubanel et al., 1991 ; Marquet, 1994). Elle se divise en trois étapes dont la première est de définir une liste de catégories représentatives des types de ligne de rivage polynésien. La seconde consiste à découper la ligne de rivage en segments homogènes assignés à une catégorie, par des observations de terrain. Les moyens de parcours de la ligne de rivage pour les relevés de terrain ont été divers : marche à pied, à terre ou sur le récif frangeant, bicyclette, kayak, ou bateau hors-bord, selon la configuration de la côte. Enfin, l'importance linéaire de chaque catégorie pour l'ensemble de l'île a été quantifiée. Les catégories retenues sont au nombre de 9. Elles sont détaillées dans le tableau 1 (dans le tableau 1, le terme « rochers » est vague, puisque les falaises sont aussi des rochers. Il serait peutêtre préférable de parler de "blocs de rochers » ou cailloutis. Il est à noter que certaines différences entre les études ont entraîné quelques difficultés de comparaison entre les îles. Ainsi, l'étude de Tahiti n'a pas concerné la totalité du trait de côte à l'instar des autres îles; de même, les deux études sur Moorea n'ont pas caractérisé les mêmes types d'anthropisations car réalisées par des opérateurs différents et avec des objectifs non similaires dans les îles Sous-le-Vent et Tahiti.

\section{Résultats}

Le tableau 2 donne la proportion de rivage anthropisé au début des années 1990:26\% à Huahine, $51 \%$ à Raiatea, $33 \%$ à Moorea, $37 \%$ à Bora-Bora, $41 \%$ à Maupiti et $47 \%$ à Tahaa. La catégorie dominante de rivage à Bora-Bora, Raiatea, Tahaa, Maupiti est artificialisée par l'homme. La catégorie dominante à Huahine est la végétation haute qui occupe $35 \%$ de la ligne de rivage. C'est la deuxième catégorie en importance après les catégories anthropisées dans toutes les autres îles sauf Moorea. À Moorea, les catégories anthropisées et les plages de sable blanc sont dominantes avec $33 \%$ d'occupation chacune. Dans les autres îles, la plage de sable blanc arrive en quatrième position, mais représente la troisième catégorie à Bora Bora, presque à égalité avec celle de la végétation haute $(17 \%)$. Les chemins de drague couvrent un maximum de $6 \%$ de la ligne de rivage sauf à Bora Bora où leur longueur atteint $10 \%$. La catégorie rochers est la moins représentée sur le rivage des îles Sous-leVent avec des valeurs inférieures ou égales à $4 \%$ dans toutes les îles sauf à Maupiti où elle représente $7 \%$ devant les chemins de drague $(6 \%)$. Deux catégories supplémentaires ont été rencontrées à Moorea, plages de sable noir dont la longueur représente $1 \%$ de la ligne de rivage en raison de l'existence de baies profondes et le beach rock qui en représente 7\% (Aubanel, 1993). Une étude similaire réalisée sur l'île de Tahiti en 2005 révèle un taux d'anthropisation proche de $30 \%$. (Leille, 2005). Les données de Tahiti ont été collectées en 2005 soit plus de onze années après les autres et cette étude n'a porté que sur une partie de l'île. Les données présentées ne sont donc pas représentatives de la totalité de l'île à l'exception des zones anthropisées puisque tous les rivages anthropisés de Tahiti ont été parcourus et leur linéaire pris en considération.

Le tableau 3 détaille la catégorie anthropisée divisée en plusieurs sous-catégories à la suite des études réalisées sur Tahiti en 2005 et les îles Sous-le-Vent en 1994. L'île de Moorea ne figure pas dans les résultats car l'anthropisation n'a pas été détaillée pour cette île. Les valeurs sont exprimées en pourcentage de longueur de ligne de rivage. La catégorie dominante pour toutes les îles, sauf Huahine, est l'anthropisation individuelle. Les valeurs les plus fortes sont trouvées à Bora-Bora : et à Maupiti avec $26 \%$ du linéaire de côte, puis à Raiatea, $25 \%$, Tahaa, $19 \%$ et Tahiti, $14 \%$. La catégorie qui vient ensuite est l'anthropisation routière qui est la plus forte à Tahaa avec $23 \%$, à Bora Bora et Raiatea $17 \%$ et pour Maupiti et Tahiti $5 \%$. Huahine présente une situation inverse avec une anthropisation routière supérieure à l'anthropisation individuelle et des valeurs qui sont respectivement de 12 et $9 \%$. L'anthropisation collective vient en troisième position dans toutes les îles excepté Tahaa: les valeurs les plus fortes se retrouvent à Tahiti (8\%), Maupiti (6\%) puis Raiatea et Bora Bora $(5 \%)$; les valeurs les plus faibles à Huahine (3\%) et Tahaa (2\%). L'anthropisation de quai ne représente jamais plus de $4 \%$ de la ligne de rivage et seulement $1 \%$ à Huahine. La catégorie marae (anciens lieux de culte polynésiens entourés généralement d'une levée de pierres volcaniques ou coralliennes) est rarement présente dans les îles Sous-le-Vent sauf à Raiatea et Maupiti où elle représente $1 \%$ de la ligne de rivage anthropisé.

Le tableau 4 présente les résultats de deux études réalisées à huit années d'intervalle sur l'île de Moorea, en 1993 par Aubanel et en 2001 par Polti. Seule cette île a fait l'objet de ce type 


\begin{tabular}{|c|c|c|}
\hline Catégories & Sous-catégories & Définition \\
\hline 1. Falaise & & $\begin{array}{l}\text { falaise ou socle rocheux de forte pente }> \\
\left.45^{\circ} \mathrm{C}\right)\end{array}$ \\
\hline 2. Rochers ou cailloutis en position naturelle & & $\begin{array}{l}\text { les rochers ont un diamètre supérieure à } 50 \\
\mathrm{~cm} \text { et les cailloutis compris entre } 5 \text { et } 50 \mathrm{~cm}\end{array}$ \\
\hline 3. Plage de sable blanc & & $\begin{array}{l}\text { au moins } 95 \% \text { de sédiments de nature coral- } \\
\text { lienne, de taille centimétrique et inférieure. } \\
\text { L'intertidal est non encombré de végétation }\end{array}$ \\
\hline 4. Plage de sable noir & & $\begin{array}{l}\text { idem que 3. avec une nature basaltique des } \\
\text { sédiments }\end{array}$ \\
\hline \multicolumn{3}{|l|}{$\begin{array}{l}\text { 5. Vasière ou zone herbacée avec éventuelle- } \\
\text { ment des palétuviers }\end{array}$} \\
\hline 6. Végétation haute & & $\begin{array}{l}\text { Substrat sablo-vaseux et détritique grossier } \\
\text { à végétation haute. }\end{array}$ \\
\hline \multicolumn{3}{|l|}{ 7. Conglomérat ou beach-rock } \\
\hline $\begin{array}{l}\text { 8. Anthropisation par murets, rochers et/ou } \\
\text { remblais, à vocation : }\end{array}$ & $\begin{array}{l}\text { 8i. individuelle } \\
\text { 8c. collective } \\
\text { 8r. de structure } \\
\text { routière } \\
\text { 8q. de quai }\end{array}$ & $\begin{array}{l}\text { Usage privé, généralement habitat } \\
\text { Usage public } \\
\text { Remblais, enrochement ou mur de soutène- } \\
\text { ment de route } \\
\text { Structure d'accostage et ses servitudes }\end{array}$ \\
\hline $\begin{array}{l}\text { 9. Chemin de drague de site d'extraction en } \\
\text { service ou abandonné. }\end{array}$ & & \\
\hline
\end{tabular}

TABlEaU 1. - Définition des différentes catégories utilisées pour caractériser la nature de rivage (d'après Marquet, 1994)

\begin{tabular}{|l|c|c|c|c|c|c|c|c|}
\hline $\begin{array}{c}\text { CATÉGORIE } \\
\text { ILLES }\end{array}$ & FALAISE & ROCHER & $\begin{array}{c}\text { PLAGE DE } \\
\text { SALEE } \\
\text { BLANCI } \\
\text { NOIR }\end{array}$ & VASE/HERBE & $\begin{array}{c}\text { VÉGÉTATION } \\
\text { HAUTE }\end{array}$ & $\begin{array}{c}\text { CONGLO- } \\
\text { MÉRAT }\end{array}$ & ANTHROPIŚE & $\begin{array}{c}\text { CHEMIN } \\
\text { DE } \\
\text { DRAGUE }\end{array}$ \\
\hline BORA-BORA & - & $3 \%$ & $17 \% \boldsymbol{I}-$ & $14 \%$ & $19 \%$ & - & $37 \%$ & $10 \%$ \\
\hline HUAHINE & $<1 \%$ & $4 \%$ & $13 \% \boldsymbol{I}-$ & $16 \%$ & $35 \%$ & $<1 \%$ & $26 \%$ & $5 \%$ \\
\hline RAIATEA & - & $2 \%$ & $6 \% \boldsymbol{I}-$ & $6 \%$ & $33 \%$ & - & $51 \%$ & $2 \%$ \\
\hline TAHAA & - & $3 \%$ & $4 \% \boldsymbol{I}-$ & $9 \%$ & $32 \%$ & - & $47 \%$ & $5 \%$ \\
\hline MAUPITI & - & $6 \%$ & $10 \% \boldsymbol{I}-$ & $18 \%$ & $19 \%$ & - & $41 \%$ & $6 \%$ \\
\hline MOOREA & - & $1 \%$ & $33 \% \boldsymbol{I}-$ & $5 \%$ & $21 \%$ & $7 \%$ & $33 \%$ & - \\
\hline
\end{tabular}

Tableau 2. - Nature de la ligne de rivages des îles Sous-le-Vent et de Moorea (Aubanel, 1993, Marquet, 1994)

\begin{tabular}{|c|c|c|c|c|c|c|}
\hline & INDIVIDUELLE & Collective & RouTE & QUAI & MARAE & TOTAL \\
\hline BORA-BORA & $26 \%$ & $5 \%$ & $4 \%$ & $1 \%$ & - & $36 \%$ \\
\hline HUAHINE & $9 \%$ & $3 \%$ & $12 \%$ & $1 \%$ & $1 \%$ & $26 \%$ \\
\hline RAIATEA & $25 \%$ & $5 \%$ & $17 \%$ & $4 \%$ & - & $51 \%$ \\
\hline TAHAA & $19 \%$ & $2 \%$ & $23 \%$ & $3 \%$ & - & $47 \%$ \\
\hline MAUPITI & $26 \%$ & $6 \%$ & $5 \%$ & $3 \%$ & $1 \%$ & $41 \%$ \\
\hline TAHITI & $14 \%$ & $8 \%$ & $5 \%$ & $3 \%$ & - & $30 \%$ \\
\hline
\end{tabular}

Tableau 3. - Nature de l'anthropisation des îles Sous-le-Vent et de Tahiti (Marquet, 1994, Leille, 2005)

\begin{tabular}{|l|c|c|}
\hline Catégorie & \% du rivage en 1993 & \% du rivage en 2001 \\
\hline Rocher & 1 & 1 \\
\hline Plage de sable blanc & 33 & 18 \\
\hline Vase/herbe & 5 & 7 \\
\hline Végétation haute & 20 & 15 \\
\hline Anthropisation & 33 & 47 \\
\hline Plage de sable noir & 1 & - \\
\hline Plage bordée de remblai & - & 4 \\
\hline Beach-rock & 7 & 7 \\
\hline
\end{tabular}

TABlEAU 4. - Évolution de la ligne de rivages de rivage à Moorea (Polti, 2001) 
d'étude qui permet d'évaluer les modifications de la ligne de rivage au cours du temps. La catégorie des zones anthropisées représente $47 \%$ de la ligne de rivage en 2001 contre $33 \%$ en 1993, les plages de sable blanc $18 \%$ contre $33 \%$. On constate également une diminution de la catégorie végétation haute qui passe de $20 \%$ en 1993 à $15 \%$ en 2001 . Les autres catégories restent stables et une nouvelle catégorie fait son apparition, celle de "plage bordée de remblai » qui représente $4 \%$ de la ligne de rivage.

\section{Discussion}

Quelques difficultés gênent une parfaite interprétation rigoureuse des données recueillies sur une quinzaine d'années. D'une manière générale, l'appréciation des diverses catégories de ligne de rivage comporte une part de subjectivité inhérente aux observateurs. Il n'en reste pas moins que ces études ont été réalisées en réponse à un besoin de connaître la nature de la ligne de rivage dans le contexte d'un développement croissant et que les résultats présentés en donnent un bon aperçu.

L'archipel de la Société qui rassemble $87 \%$ de la population de la Polynésie française présente de manière générale une anthropisation de ses rivages assez importante bien que variable d'une île à l'autre. En 1993, la moyenne pour les îles Sous-le-Vent se situe à $40 \%$ et à $33 \%$ pour Moorea. L'île au rivage le moins artificialisé est Huahine avec seulement $26 \%$ du rivage modifié par l'homme. En 2005, l'anthropisation de l'île de Tahiti est de $30 \%$. Tahiti est pourtant la plus peuplée de ces îles, mais ce faible taux d'anthropisation tient à la présence de nombreuses côtes escarpées sur la presqu'île et la côte est qui sont peu propices à la présence de l'homme et au développement de ses activités compte tenu d'une ouverture totale sur l'océan (le récif barrière est submergé) et de l'absence de lagon.

La comparaison des observations réalisées en 1993 et en 2001 à Moorea donne un aperçu de l'évolution de la ligne de rivage pendant ces quinze dernières années. L'anthropisation y a fortement augmenté, + $14 \%$ entre 1993 et 2001. Cette augmentation s'est semble-t-il faite au détriment des plages de sable blanc dont la proportion est passée de 33 à $18 \%$ alors que les autres catégories sont restées stables. L'apparition d'une catégorie " sable blanc + remblais ", équivalant à $4 \%$ de la ligne de rivage, semble être une phase transitoire entre les catégories " plage de sable blanc » et « anthropisation » et il est fort probable qu'aujourd'hui, ces $4 \%$ ont fait passer l'anthropisation de la ligne de rivage à Moorea au-delà des $50 \%$. C'est l'anthropisation individuelle, liée à l'habitation privée, qui est essentiellement responsable de cette augmentation (Polti, 2001). Gros (1995) a étudié dans le district d'Arue à Tahiti la motivation, les procédures et les réalisations de ces remblais par les riverains pour conclure que leur mise en place obéissait non seulement à un souhait de protection mais aussi à un gain en terres sur le lagon. L'accroissement de la population sur Moorea, île très proche de Tahiti, associé à une envolée du prix des terrains expliquerait cette augmentation. Aubanel (1993) avait calculé le coût moyen d'un mètre carré de remblais par rapport à un mètre carré de terrain en bord de mer. Ces coûts étaient respectivement de 1500 et $15000 \mathrm{~F} \mathrm{CPF}$, soit un coût pour un terrain gagné sur le lagon dix fois plus élevé que la mise en place du remblai. Les remblais sont toutefois interdits, mais les réglementations ne sont pas toujours respectées et les contrevenants rarement condamnés. Les prix ayant fortement augmenté depuis deux décennies, on peut penser que le remblai est encore plus avantageux à l'heure actuelle. Il y a aussi une évolution des mentalités qui pourrait expliquer l'augmentation des remblais au détriment des plages de sable blanc: Les habitants ne supportent plus de gérer un terrain "ouvert» et de constater que leur plage est accessible à la libre circulation. Ils éprouvent le besoin de clôturer leur propriété et préfèrent renoncer à la jouissance d'une portion de plage et construire un remblai jusqu'au bord de l'eau, pour éviter tout passage.

La proximité de Moorea avec Tahiti fait de la première un cas un peu particulier puisque plusieurs résidents à Moorea ont leur activité professionnelle à Tahiti. Les moyens de transport entre les deux îles se sont d'ailleurs considérablement améliorés et le centre de Papeete n'est plus qu'à une demi-heure de bateau de Moorea alors que les routes pour rejoindre Papeete depuis les communes alentour sont de plus en plus congestionnées. Moorea est devenue une banlieue de Tahiti et a vu sa population croître en conséquence. Dans le cas des îles Sous-le-Vent, le taux d'anthropisation a sans doute augmenté, mais en moindre mesure en comparaison avec Moorea.

Dans les îles Sous-le-Vent, Marquet (1994) avait vainement essayé de mettre en évidence des facteurs responsables de l'anthropisation, notamment la densité de population et le développement économique. Le facteur géomorphologique est à considérer dans l'évolution de l'artificialisation, particulièrement l'anthropisa- 
tion à caractère individuel qui est essentiellement réalisée aux dépens de couverts de végétation haute. Les rochers s'accompagnent le plus souvent d'une bande littorale au relief peu propice à l'habitat. Les plages présentent une valeur économique importante et sont plutôt préservées, même si Moorea est un contre-exemple. Enfin, les vasières constituent un substrat peu adéquat à l'implantation humaine et au développement d'activités sans travaux d'envergure. La détermination d'une catégorie de ligne de rivage en un point donné obéit à un processus complexe d'interactions de facteurs naturels et humains.

L'artificialisation de la ligne de rivage par mise en place de remblais et de murets de protection, qui réduisent la plage quand ils ne la suppriment pas, engendre des effets néfastes sur l'environnement. Le premier est un effet physique d'accélération de l'érosion de la côte par augmentation de la turbulence par forte houle, induisant l'entraînement irréversible des sédiments jusqu'à la disparition de la plage (Paskoff, 1994). Enfin, les ouvrages de remblais réduisent des zones frangeantes alors que des recherches ont montré que ces dernières étaient des zones de nurseries pour les juvéniles de poissons (Salvat, 1987 ; Galzin, et al., 1989 ; Lefèvre, 1991). Les études menées sur les dégradations anthropiques des récifs frangeants et leurs incidences sur les peuplements ichtyologiques ont en effet révélé une étroite corrélation entre le faible taux de recrutement en larves de poissons et l'état de dégradation d'un milieu (Galzin et al., 1989). Le sud de l'île de Moorea où la présence humaine est faible présentait le meilleur taux de recrutement de poissons, en rapport avec la stabilité du récif frangeant. À l'opposé, les secteurs nord et est de l'île beaucoup plus habités avaient des taux de recrutement médiocres.

Signalons enfin que le fort accroissement de la population entraîne des rejets d'eaux usées plus importants qui viennent enrichir les lagons en nutriments; ces derniers entraînent la prolifération de macro-algues qui entrent directement en compétition avec les coraux (Payri et Naim, 1982) et présentent également un terrain favorable au développement de foyers ciguatériques, véritable problème de santé publique dans certaines îles de la région (Bagnis et al., 1974)

Devant les menaces pesant sur le patrimoine naturel de la Polynésie française, le gouvernement s'est doté d'un arsenal réglementaire destiné à contrôler l'extraction des granulats coralliens ainsi que l'occupation du domaine maritime. Les récifs coralliens étaient bien protégés par ces réglementations, mais leur application sur le terrain laissait fortement à désirer : faiblesse des actions d'informations sur la réglementation, absence de surveillance et de contrôle, en partie par manque de moyens, absence d'intervention, dérogations très fréquentes. À titre d'exemple, les dragages dans le récif pour pourvoir aux besoins en granulats, pour la construction de routes en particulier, ou pour la réalisation d'aménagements maritimes comme les ports ou les chenaux de navigation, ont été l'une des causes majeures de destruction des récifs pendant de longues années dans les îles en développement de l'archipel de la Société. Il y a trente ans les granulats coralliens constituaient quasiment la seule source de matériaux du Territoire. Aujourd'hui encore, malgré l'interdiction d'extraire du corail depuis 1984, le statut dérogatoire perdure depuis vingt-cinq ans et le matériau corallien demeure la source majeure de granulats. En 1993, aux îles Sous-le-Vent, la surface totale de récif frangeant directement détruite par les extractions au cours des années passées a été estimée à 102 ha $(3,5 \%)$ et, compte tenu de la perturbation des récifs voisins, environ 7 à $11 \%$ des récifs frangeants en ont subi l'impact (Marquet, 1994).

À la fin des années 1990, les premiers « Plans de gestion de l'espace maritime (PGEM) » sont mis en chantier dans le cadre réglementaire du code de l'Aménagement de Polynésie française. Ils sont une adaptation des « Schémas de mise en valeur de la mer (SMVM) » lancés en 1983 en France métropolitaine. Ces PGEM sont élaborés au cours de longues concertations entre toutes les catégories d'usagers aux intérêts divergents et sont censés aboutir à un consensus. Ils se présentent sous la forme d'un document d'urbanisme réglementant les conditions d'utilisation des lagons et des façades maritimes et l'exploitation des ressources, en fonction de la vocation des différentes zones lagonaires (pêche, conservation, tourisme, navigation etc.). La responsabilité de l'élaboration des PGEM est du ressort des ministères de l'Aménagement, de la Mer, et de l'Environnement. Le PGEM de Moorea a été rendu exécutoire par l'arrêté $\mathrm{n}^{\circ} 410 / \mathrm{CM}$ du 21 octobre 2004 (parution au Jo du 22 octobre 2004). Les rivages de Moorea sont désormais protégés des remblais lorsqu'ils ne sont pas d'utilité publique et de l'extraction de granulats coralliens, sauf en cas de réhabilitation de sites, de protection d'ouvrages publics ou de construction de marinas et, en tout état de cause, après une étude d'impact sur l'environnement. Le PGEM de la commune de Fakarava regroupant 7 atolls des Tuamotu a également été adopté dans le cadre d'une Réserve de la Biosphère du programme MAB de l'UNESCO. Mais dans ces îles 
basses où les pressions démographiques sont très faibles il n'y a aucun problème d'artificialisation de la ligne de rivage du lagon qui correspond presque partout à des plages de sable blanc; mais il y a des occupations du domaine public maritime par des familles dont les terrains sont en indivision compte tenu de la situation foncière en Polynésie française.

\section{Conclusions}

Ces premières études sur la dynamique des lignes de rivage ont montré que le phénomène d'anthropisation des lignes de rivage était plus ou moins avancé selon les îles. Dans les années 1990, la part de rivage anthropisé était comprise entre 25 et $50 \%$ de la longueur totale du rivage. Les côtes modifiées par l'homme se retrouvent maintenant prépondérantes dans la majorité des îles. La modification du littoral à des fins d'habitat privé est la principale cause de cette artificialisation. La tendance évolutive de ce phénomène, telle qu'elle est illustrée à Moorea, est à la forte augmentation. Les facteurs d'anthropisation sont à la fois démographiques, économiques et géomorphologiques. Les impacts écologiques de ce phénomène ne sont pas négligeables, érosion accélérée, diminution du recrutement des poissons juvéniles, eutrophisation des lagons en raison de la croissance démographique et du rejet de nutriments. Pendant de nombreuses années, la réglementation a été très souple face à l'utilisation du littoral mais devant l'accentuation du phénomène d'anthropisation, les pouvoirs publics se sont dotés d'un arsenal réglementaire et très récemment, un premier plan de Gestion de l'espace maritime a été mis en place à Moorea. Il serait judicieux que les autres îles, notamment les îles hautes de la Société, se dotent de PGEM analogues et qu'une attention particulière soit portée au maintien de la ligne de rivage lorsqu'elle est encore naturelle.

D'autres mesures pourraient être envisagées favorisant la protection non seulement de la ligne de rivage naturelle mais aussi celle du littoral côtier. L'exemple du Conservatoire du Littoral créé en France métropolitaine en 1975 mérite d'être considéré en vue d'une éventuelle application en Polynésie française ou de la création d'une structure analogue. On doit se réjouir des résultats du Conservatoire en métropole : protection de $1030 \mathrm{~km}^{2}$ sur plus de $800 \mathrm{~km}$ de rivage (IFEN, 2007).

\section{BIBLIOGRAPHIE}

Aubanel A., 1993. Valeurs socio-économiques du milieu corallien récifal et de ses ressources : application à une île océanique du Pacifique Sud : Moorea, archipel de la Société, thèse de doctorat de l'université Michel de Montaigne-Bordeaux III, 320 p.

Aubanel A, Bonvallot J. et Loubersac, 1991. Du trait de côte du littoral corallien d'une île tropicale en voie de développement (Moorea, Polynésie française), Le littoral, ses contraintes environnementales et ses conflits d'utilisation (colloque 1991), Nantes, Union des Océanographes de France, Société d'écologie, $4 \mathrm{p}$.

Aubanel A, Marquet, Colombani et Bernard SALVAT, 1999. Modifications of the shore line in the Society islands (French Polynesia) Ocean Coastal Management 42, pp. 419-438.

Bagnis R., Denizot M., Drollet J. et Laigret J., 1974. Biotopes ciguatérigènes en Polynésie française, Revue internationale d'Oceanographie médicale 35/36, pp. 213-223.

GABrIE C., 1988. Environnement Huahine. Synthèse des données environnementales, recommandations, rapport antenne EPHE/Museum, Moorea (Polynésie française), CRIOBE 1988 RA 24, 213p.

Galzin R., Baldwin J., Chauvet C., FontaineVernaudon Y., Gabrie C., Holthus P., Payri C. et Planes S., 1990. Étude du lagon de Bora-Bora en vue de la création d'un parc marin. Action Délégation à l'environnement, rapport antenne EPHE/Museum, Moorea (Polynésie française), 193p.

Galzin R, C. Gabrie, Lefevre et Bernard Salvat, 1989. Dégradations anthropiques des zones frangeantes des récifs coralliens polynésiens, influences sur l'ensemble de l'écosystème, incidences sur les peuplements coralliens, Action, $51 \mathrm{p}$.

Grellet B., Combes P., et Duclerq A., 1993. Étude des risques d'érosion de la pointe Matira à BoraBora (Îles Sous-le-Vent, Polynésie française) et moyens de lutte, geo-ter, direction de l'assistance technique, délégation à l'Environnement, rapport $\mathrm{n}^{\mathrm{o}}$ GTR/DAT/1293-20, $56 \mathrm{p}$.

Gros S., 1995. Les remblais : étude des motivations, procédures et réalisations. L'exemple d'Arue (Tahiti, Polynésie française), Diplôme d'études approfondies « Connaissance et gestion des milieux coralliens littoraux et océaniques $»$, rapport CRIOBE - EPHE et service de l'Urbanisme, 33 p.

FEN, 2007. Le littoral, entre nature et artificialisation croissante, Institut français de l'Environnement 120, pp. 1-4.

LefÈvre A., 1991. Dynamique de l'installation des juvéniles de poissons sur les récifs coralliens (Moorea, Polynésie française), thèse de doctorat de l'université Paris VI, 33 p.

LeILle L., 2005. Caractérisation de la ligne de rivage de l'île de Tahiti, Polynésie française, Moorea (Poly- 
nésie française), rapport antenne EPHE/Museum, CRIOBE, $2005 \mathrm{RA}: 13 \mathrm{p}$.

MARQuet N., 1994. La ligne de rivage des Îles Sousle-Vent (Archipel de la Société, Polynésie française), catégorisation et quantification, taux d'anthropisation, DEA de l'université française du Pacifique, $31 \mathrm{p}$.

PASKOFF R., 1994. Les littoraux, impact des aménagements sur leur évolution, Paris, Masson, coll. Géographie, $254 \mathrm{p}$.

PAYRI C. et NAIM O., 1982. Variations entre 1971 et 1980 de la biomasse et de la composition de macroalgues sur le récif corallien de Tiahura (île de Moorea, Polynésie française). Cryptogamie et Algologie , 3 (3), pp. 229-240.
Polti S., 2001. Caractérisation de la ligne de rivage et du domaine maritime de l'île de Moorea, Polynésie française, Moorea (Polynésie française), rapport antenne EPHE/Museum, CRIOBE, 2001 RA 97, 26 p.

RaYNAL J.B., 2004. Le littoral de Taiarapu Ouest, un espace en mutation ?, mémoire de maîtrise de l'université de la Polynésie française- Paris IV Sorbonne.

Salvat Bernard, 1987. Human impacts on coral reef: facts and recommendations, Moorea (Polynésie française), antenne de Tahiti Museum/ EPHE, $253 \mathrm{p}$.

SinDJOUN G., 2002. Étude en 2001 de la ligne de rivage de Moorea, Brest, université de Bretagne occidentale, Institut de géoarchitecture, rapport de stage au service de l'Urbanisme, $25 \mathrm{p}$. 